\title{
Detection of Blast Cells in Microscopic Images Using Optimization Algorithms
}

\author{
M.Venkata Dasu, P.Subbaiah, K.Srilatha
}

\begin{abstract}
Now a day's in medical field, the most prevalent and hazardous disease is blood cancer. It starts in bone marrow where the blood is produced and prevents many of its regular functions. At an approximation for every three minutes one person in the world is diagnosed with the blood cancer. Early detection of the cancer is necessary for the proper treatment, so as to save the lives of mankind. In general the blood cancer diagnosis will be performed by visual examination of the blood samples of the patient under microscope. The blood cancer detection accuracy of this method depends on the technical skilling abilities of the operator and often leads non-standardized report. To improve patient diagnosis various image processing methods are developed to extract useful information from microscopic images. This could help Hematologists in their diagnostic process.

In this paper pre-processing and post-processing methods are applied on microscopic images. These images will be acquired from either public or private database. In this work the images were collected from Microscopyu which is a public database. Pre-processing methods involves color conversion i.e. RGB to grayscale, removal of noise by median filter and an improved contrast enhancement technique CLAHE is implemented. Later Post-processing methods are applied. In this stage Otsu segmentation and optimization algorithms are combined for improving segmentation accuracy. Optimization algorithms used are Particle Swarm optimization (PSO) and Cuckoo Search algorithms (CSO) and Finally features of the segmented image can be extracted from d Scale Invariant Feature Transform (SIFT) . In this work existing method is PSO and proposed method is CSO. At the end the qualitative analysis of the work is done through the statistical parameters like segmentation accuracy, sensitivity, specificity, PSNR and CPU time.
\end{abstract}

Index Terms-Leukemia, Enhancement, ACO, PSO, SIFT.

\section{INTRODUCTION}

The basic element of human life is blood which delivers required substances such as oxygen and nutrient to the cells and removes metabolic wastage from the cells. Human body contains 5 liters of blood. Blood performs many functions in the body human .It regulates body temperature and body $\mathrm{PH}$ value. The main components of blood is white blood cells (WBC) ,Red blood cells (RBC) and Platelets[1]. White Blood cells are also called as leucocytes they originate from bone marrow. They improve immunity power in the body by fighting against bacteria, viruses and other foreign organisms. Red blood cells are called as erythrocytes and are crucial for the exchange of oxygen and carbon dioxide in the

Revised Version Manuscript Received on 10 September, 2019.

M.VenkataDasu, Research Scholar, Dept. of ECE, Rayalaseemauniversity, Kurnool, Andhra Pradesh, India.

(Email: dassmarri@gmail.com)

Dr. P.Subbaiah, Professor of ECE, NallaNarasimhareddy Engineering College, Ghatkesar, Hyderabad, Telangana State, India.

(Email: subbaiah.nani45@gmail.com)

K.Srilatha, Assistant professor of CSE, NNRG,Hyderabad, Telangana,India. body. Platelets are responsible for clot the blood to heal the wounds and prevent the excess blood loss.

Blood cancer is also called as Leukemia, it is a disorder in blood cells and commonly effects the white blood cells or leucocytes [2]. The reason for blood cancer is overproduction of white blood cells and irregularity in the shape. Early detection of cancer is required to save the lives. In general pathologists diagnose the cancer by examining blood samples under microscope. But this investigation has many disadvantages such as time consuming, and less accuracy. Accuracy depends on operator skills [3-5]. In this paper a dynamic method was developed for automatic detecting and segmentation of blast cells from normal cells (WBC) in microscopic images[6]. This combined uses the image processing methods and optimization algorithms.

In this work a pre-processing and post-processing approaches are present for detection of blast cell (cancerous cell) from normal White blood cells. In this research work three kinds of preprocessing methods are implemented such as the color conversion technique for RGB to gray scale and second Pre-processing method is de-noising using median filter. Finally it is required to enhance the proposed image by using CLAHE method

Otsu segmentation is used to detect blast cells and then Particle swarm optimization (PSO) and Cuckoo Search Algorithms(CSO) are applied on segmented image in order to improve the segmentation accuracy. In this work Particle swarm optimization (PSO) is existing method and Cuckoo Search Algorithms (CSO) is proposed method.

The organization of this technical paper is structured as follows.Chapter. 2 describes the methodology of the proposed method. Chapter. 3 depicts the investigation of experimental results with statistical parameters and finally Chapter.4 designates the conclusion of the work.

\section{METHODOLOGY}

The objective of the proposed method is to isolate blast cells from normal blood cells. It contains two parts preprocessing and post processing. Initial step is the image acquisition and in this work images are collected from public database such as Microscopyu. pre-processing is the second step where the steps are color conversion i.e color image converted into gray scale image followed by filtering process using Median filter to remove the noise and CLAHE is applied to enhance the contrast of an image. Third step is detecting of blast cells using Otsu segmentation is done and 
followed by optimization algorithm for improving segmentation algorithm. Final step is feature extraction where segmented image feature are extracted using Scale Invariant Feature Transform (SIFT).

Block diagram of the proposed model is shown in figure1.The working of each module is explained in this section.

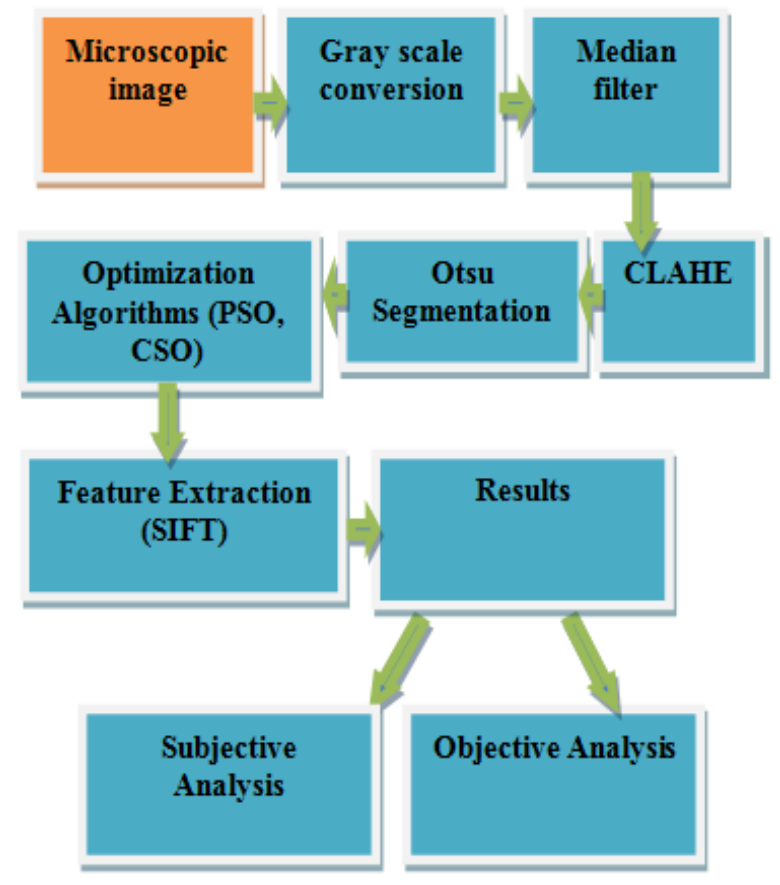

Figure1.Block Diagram of Proposed model

\section{A. Image acquistion}

An input microscopic image for this work is considered from public database.

\section{B. Color conversion}

In general the images(Microscopic Images) are available for the processing as raw data. Therefore the microscopic images are to be converted into a gray scale image format..

\section{Median Filter}

In order to improve the results of the later processing it is essential to mitigate the noise and noise removal is typical preprocessing step. In this work noise is removed by a nonlinear filter called Median filter. It is widely used in image processing because it preserves the while removing noise. It can be accomplished by sliding a $3 * 3$ mask over the image and calculate the median value and center of the mask is replaced by median filter. Generally the size of the masks are odd .if $c=\left[c_{1}, c_{2}, c_{3}, c_{4}, \ldots . . c_{9}\right.$. are the pixel values .

Median value can be calculated as

$$
\text { Median }(\mathrm{c})=\mathrm{c}+1 / 2 \text {. }
$$

\section{CLAHE (Contrast Limited Adaptive Histogram} Equalization)

It is a contrast enhancement technique which was developed to prevent over amplification of noise. It works on smaller regions of an image not on entire image. These small regions are called as tiles. The flowchart of CLAHE shown in figure2.

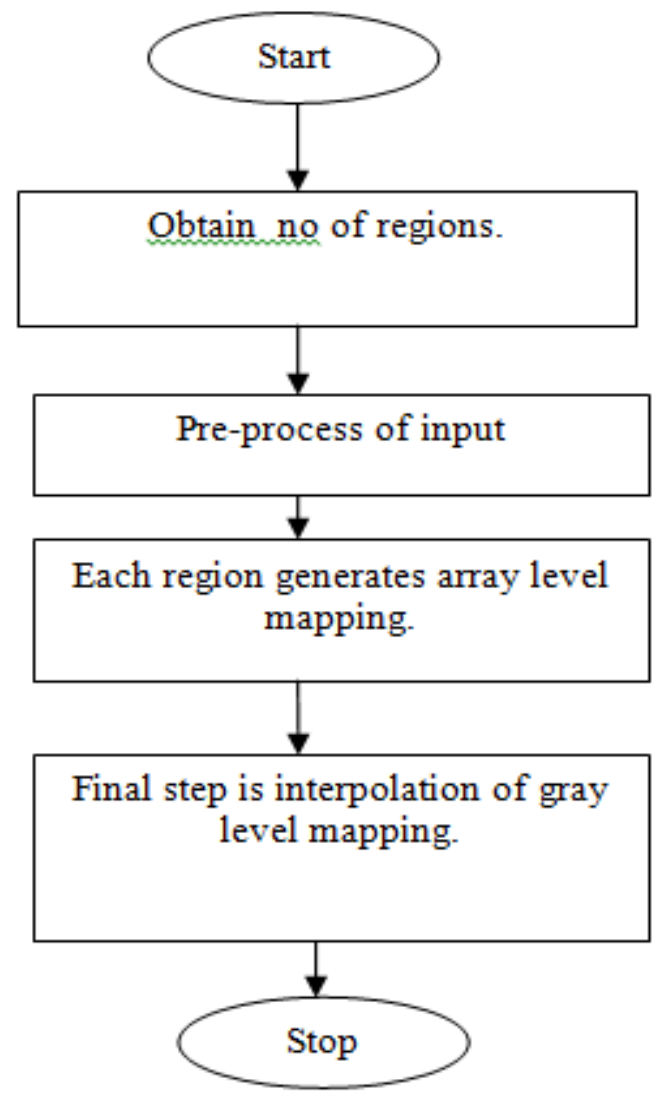

Figure 2.Flow chart of CLAHE.

\section{E. Otsu segmentation}

The very fundamental step in digital image processing is segmentation. It acquires many prominent improvements in image processing. In terms of it is the process of separating or dividing the image in to meaningful objects or regions. In the proposed model Otsu segmentation is presented. It directly operates on bimodal histogram, so it is fast.

Algorithm:

1. Estimate of the intensity level and compute histogram.

2. Setup initial $\mathrm{w}_{\mathrm{i}}(0)$ and $\mu_{\mathrm{i}}(0)$.

3. Measure the threshold value for all possible values $\mathrm{t}=1$....maximum intensity

I. Update $\mathrm{w}_{\mathrm{i}}$ and $\mu_{\mathrm{i}}$.

II. Compute $\sigma_{b}^{2}(t)$.

4. Choose the desired threshold corresponds to maximum variance $\sigma_{b}^{2}(\mathrm{t})$.

5. Desired threshold $=\sigma_{b 1}^{2}(t) .+\sigma_{b 2}^{2}(t) / 2$.

\section{OPTIMIZATION ALGORITHMS}

Optimization is used in many applications such as health care, production and image processing etc. optimization is maximize or minimize the one or more objective functions.

\section{A. Particle swarm Optimization (PSO)}

PSO is inspired by social behavior of bird flocking or bird schooling. it was first described by James Kenned and Russel.C.Eberhart. It is a Artificial Intelligence algorithm and can be used find optimum solutions to the difficult problem[8]. It is simple algorithm and easy to implement

- PSO is analyzed with a group of random particles and

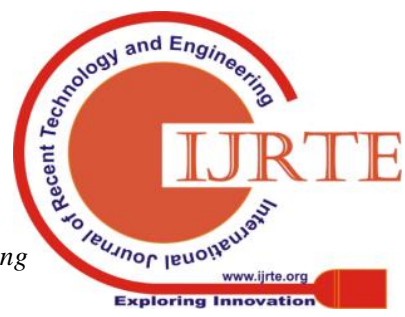


then searches for optimal by updating generations.

- Particle moves through the solution space and they are investigated for a specific fitness criteria periodically.. The $p_{\text {best }}$ and $g_{\text {best }}$ values are expected to updated unconditionally for every iteration

- Every particle sample tries to alter their velocity and position based on $p_{\text {best }}$ the distance between its current position and distance between its current position and $\mathrm{g}_{\text {best }}$.

The original velocity update equation is

$$
A_{i}^{t+1}=A_{i}{ }^{t}+\phi_{1} \cdot r_{1}\left(p_{i}-Y_{i}{ }^{t}\right)+\phi_{2} \cdot r_{2}\left(p_{g}-Y_{i}^{t}\right)
$$

Here $\phi_{1}$ and $\phi_{2}$ are Acceleration constants.

The Position update equation is $\mathrm{Y}_{\mathrm{i}}^{\mathrm{t}+1}=\mathrm{Y}_{\mathrm{i}}^{\mathrm{t}}+\mathrm{A}_{\mathrm{i}}^{\mathrm{t}+1}$

\section{B. Cuckoo Search Optimization (CSO)}

In the year 2009 the scientists Yang and Deb were invented Cuckoo search optimization algorithm. It was inspired by the eggs in the nest of host bird. It is based on three rules[9 -10].

- Each Cuckoo lays an egg at a time and leaves their eggs in the nest which is chosen randomly by Cuckoo birds. carrying over to the next generation.

- In fixed number of available nests a host can discover a alien egg with a probability p. In this case the host bird can through the egg away or built a new nest.

Algorithm steps

1. create $n$ host nest initial population.

2. Lay the egg $\left(a p^{1}, b p^{1}\right)$ in $p$ nest

Where ap ${ }^{1}=a p+\operatorname{randomwalk}($ Levy flight $)$ ap

$\mathrm{bp}=\mathrm{bp}+$ randomwalk(Levy flight) $\mathrm{bp}$

3. Collate Cuckoo's egg fitness with the host egg fitness

4. If fitness of Cuckoo's egg is superior than host egg, swap the egg by Cuckoo's egg in p nest

5. If it find the host bird then nest is discarded and fresh one is constructed. Iterate step 2 to 5 up to criteria of termination is fulfilled [11-14].

\section{SIFT(Scale Invariant Feature transform):}

It is a computer vision technique which portrays the local features of an image. It was proposed by David Lowe. It involves 4 steps such as orientation assignment, scale space extrema, key point descriptor and localization.[15].

- Orientation assignment:calculate finest orientation(s) for every key point area.

- Scale space extrema:Explore numerous image locations and scales.

- Key point descriptors:It investigates every key point area at selected scale by using local image gradients.

- Key point localization: Depends on stability it selects key points and it fit a frame model to find the location.

\section{EXPERIMENTAL RESULTS}

The input microscopic image is shown in figure1.which is in color format for easy processing the image is converted in to gray scale image. The size of the image which has taken for this work is $128 * 128$. obligate brood parasitism of Cuckoo species by laying their

- The nest which contains best or quality eggs are

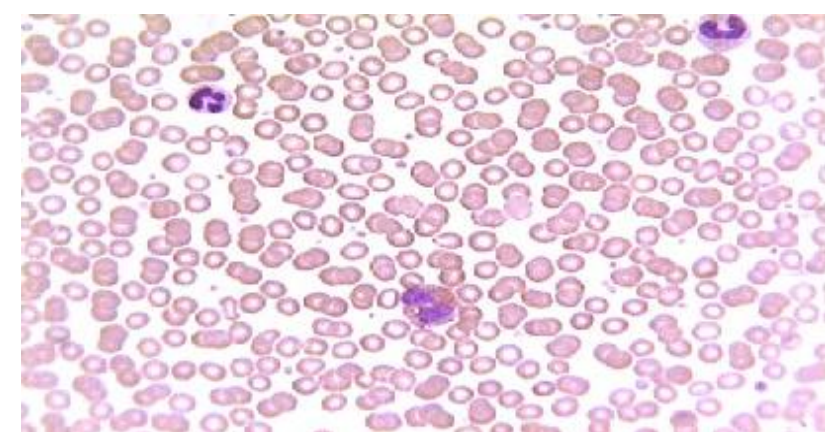

Figure 1. Input Microscopic image.

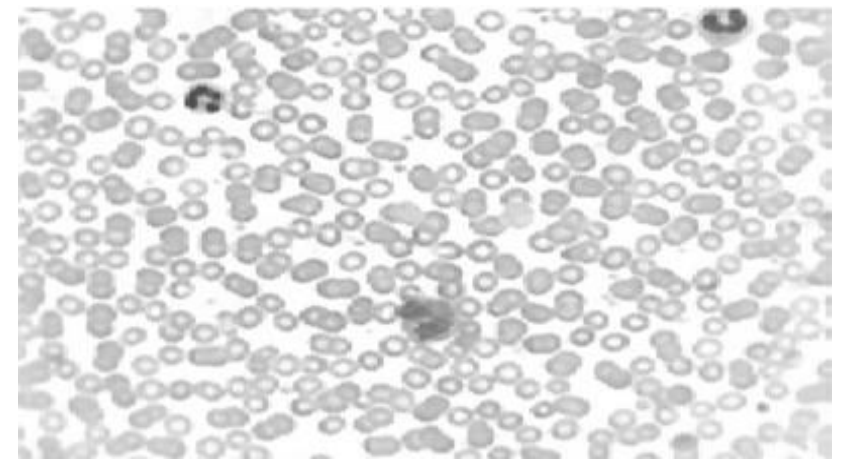

Figure 2.Median Filtered image.

The figure 2 sows the median filtered image where noise can be removed in the microscopic image. Noise is the basic limitation in the images.

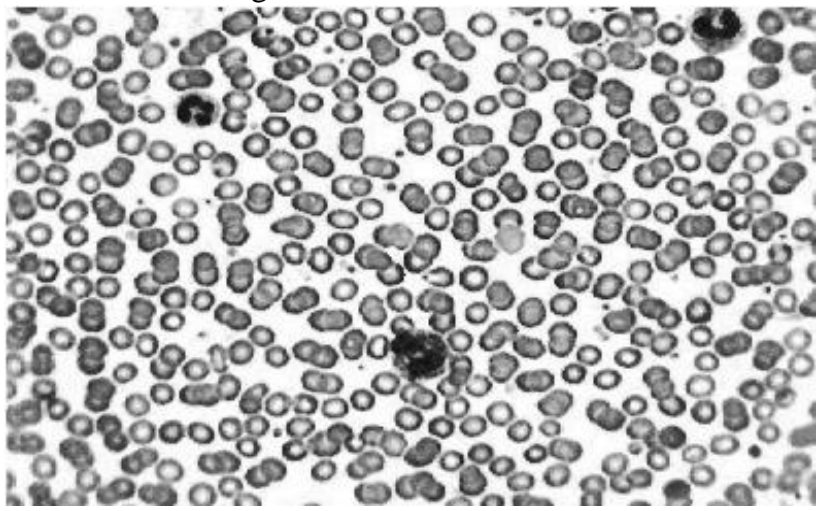

Figure 3.CLAHE image.

Figure 3 shows the contrast improvement using CLAHE. It makes the edges of white blood cells sharper.

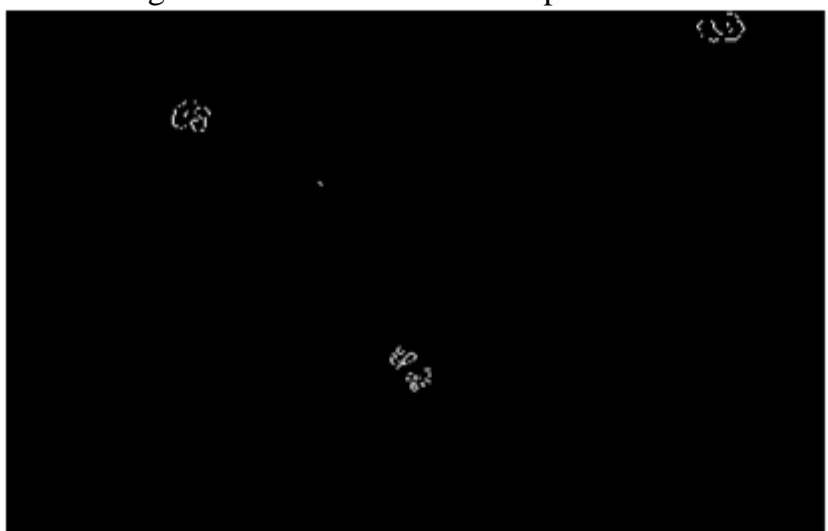

Figure 4.PSO image.

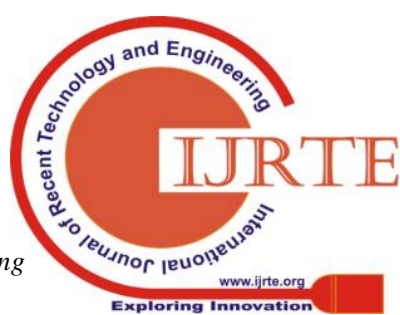


The figure 4 and 5 shows the segmented image with PSO and CSO .Separation of blast cell from normal white blood cell is difficult in PSO. In CSO malignant cells boundaries are extracted .

Table 1.Statical parameters

\begin{tabular}{|c|c|c|c|c|}
\hline S.No & Parameter & ACO & PSO & CSO \\
\hline 1 & PSNR & 24.90 & 28.34 & 48.08 \\
\hline 2 & ACCURACY & 28.58 & 52.18 & 98.91 \\
\hline 3 & SENSITIVITY & 34.03 & 53.15 & 91.01 \\
\hline 4 & SPECIFICITY & 25.36 & 43.14 & 62.35 \\
\hline 5 & FPR & 41.79 & 47.5 & 38.13 \\
\hline 6 & CPU Time (sec) & 18 & 12 & 10 \\
\hline
\end{tabular}

The results are analyzed in this work are subjective analysis and objective analysis. Objective analysis includes statistical parameters. In this work the parameters are considered are segmentation accuracy, sensitivity, specificity, FPR and CPU processing time.

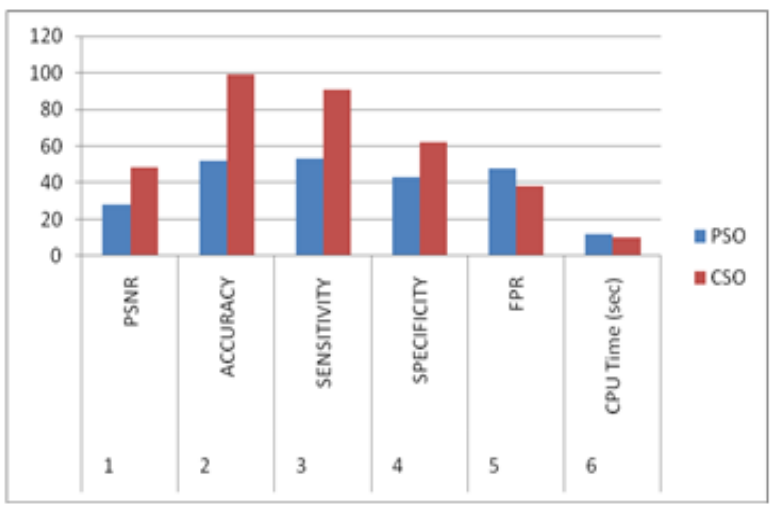

Figure 5.Graphical Representation of parameters.

Figure 5 represents the graphical representation of the parameters. It shows that the proposed method CSO has better values than existing method PSO.

\section{CONCLUSION}

In this paper amalgamation of Otsu segmentation and optimization algorithms are utilized to separate blast cells from normal cells in the microscopic blood images. Here PSO is existing and CSO is proposed method. The segmentation accuracy and other statistical parameters obtained are better for proposed method than existing method.

\section{ACKNOWLEDGEMENT}

The first author is a research scholar in Rayalaseemauniversity, Kurnool and he would like to thank Rayalaseema university and Annamacharya Institute of technology and sciences, Rajampet for providing research facilities to carry out this research work.

\section{REFERENCES}

1. S.S.Savkare,S.P.Narote,"Blood cell segmentation from Microscopic blood images" in international conference on information processing.P.no.502-505,2015.
2. Dorini, LeyzaBaldo, Rodrigo Minetto, and Neucimar J Leite. "Semiautomatic white blood cell segmentation based on multiscale analysis," IEEE journal of Biomedical and Health Informatics, vol. 17,No.1, pp 250-256, 2013.

3. C. Y. Lin, M. Wu, J. A. Bloom, I. J. Cox, and M. Miller, "Rotation, scale, and translation reilient public watermarking for images," IEEE Trans. Image Process., vol. 10, no. 5, pp. 767-782, May 2001.

4. Man Yan, JianyongCai, JiexingGao, LiliLuo, "K-Means cluster algorithm based on color image enhancement for cell segmentation," in Proc. on Biomedical Engineering and Informatics (BMEI), IEEE, pp 295-299, 2012.

5. RongtaiCai, Qingxiang Wu, Rui Zhang, Lijuan Fan, ChengmeiRuan,"Red blood cell segmentation using Active Appearance Model," in Proc. on Signal Processing (ICSP), vol. 3, pp. 1641-1644. IEEE, 2012.

6. Abbas, Naveed, and DzulkifliMohamad, "Microscopic RGB ColorImages Enhancement for Blood Cells Segmentation in $\mathrm{YCbCr}$ Color Space for K-Mean Clustering", Journal of Theoretical \& AppliedInformation Technology, vol. 54, no. 1, 2013.

7. Xin-She Yang, Suash Deb, "Cuckoo search: recent advancesand applications", Springer-verlog, London, 2013.

8. M.VenkataDasu, P.Subbaiah,'Leukemia detection in microscopic imagery using optimization algorithm" in IJET,pp.299-303,2018.

9. C.Venkatesh, L.SivaYamini,"A Novel optimization for detection of foot ulcers on Infrred images" in international conference on recent trends in electrical, electronics and computing technology",pp.41-43, 2017.

10. Srishti, "Technique Based on Cuckoo's Search Algorithm for Exudates Detection in Diabetic Retinopathy", Ophthalmology Research: An International Journal2 (1): 43-54, 2014.

11. Xin-She Yang, Suash Deb, "Cuckoo search: recent advances and applications", Springer-verlog, London, 2013.

12. AbdesslemLayeb, “A novel quantum inspired cuckoo search for Knapsack problems”, International Journal of Bio-Inspired Computation Vol. x, No. x, 2011.

13. H. Zheng and Y. Zhou, "A Novel Cuckoo Search Optimization Algorithm Base on Gauss Distribution", Journal of Computational Information Systems 8: 10, 4193-4200, 2012.

14. Xin-She Yang, "Cuckoo Search and Firefly Algorithm",Springer Press, 2014.

15. M.VenkataDasu, P.Subbaiah,"Leukemia detection of leucocytes in microscopic imagers with swarm intelligence algorithms" in Helix scientific journal Vol.9(1),pp.no:4838-4842,2019 